\title{
Alkaloid Biosynthesis in the Early Stages of the Germination of Argemone mexicana L. (Papaveraceae)
}

\author{
Jorge Xool-Tamayo $\mathbb{D}^{\mathrm{D}}$, Yahaira Tamayo-Ordoñez, Miriam Monforte-González, José Armando Muñoz-Sánchez \\ and Felipe Vázquez-Flota *(i)
}

check for

updates

Citation: Xool-Tamayo, J.;

Tamayo-Ordoñez, Y.;

Monforte-González, M.;

Muñoz-Sánchez, J.A.; Vázquez-Flota,

F. Alkaloid Biosynthesis in the Early

Stages of the Germination of

Argemone mexicana L. (Papaveraceae).

Plants 2021, 10, 2226. https://

doi.org/10.3390/plants10102226

Academic Editor: Vincent

Courdavault

Received: 21 September 2021

Accepted: 11 October 2021

Published: 19 October 2021

Publisher's Note: MDPI stays neutral with regard to jurisdictional claims in published maps and institutional affiliations.

Copyright: (c) 2021 by the authors. Licensee MDPI, Basel, Switzerland. This article is an open access article distributed under the terms and conditions of the Creative Commons Attribution (CC BY) license (https:/ / creativecommons.org/licenses/by/ $4.0 /)$.
Centro de Investigación Científica de Yucatán, Unidad de Bioquímica y Biología Molecular de Plantas, Calle 43, No. 130 Chuburná, Mérida 97205, Mexico; jxool@cicese.mx (J.X.-T.); yahairatamayo@uadec.edu.mx (Y.T.-O.); mmg@cicy.mx (M.M.-G.); arms@cicy.mx (J.A.M.-S.)

* Correspondence: felipe@cicy.mx; Tel.: +52-(999)-942-8330

\begin{abstract}
The synthesis of the benzylisoquinoline alkaloids, sanguinarine and berberine, was monitored in Argemone mexicana L. (Papaveracea) throughout the early stages of its hypocotyl and seedling development. Sanguinarine was detected in the cotyledons right after hypocotyl emergence, and it increased continuously until the apical hook unbent, prior to the cotyledonary leaves unfolding, when it abruptly fell. In the cotyledonary leaves, it also remained at low levels. Throughout development, berberine accumulation required the formation of cotyledonary leaves, whereas it was quickly detected in the hypocotyl from the time it emerged. Interestingly, the alkaloids detected in the cotyledons could have been imported from hypocotyls, because no transcriptional activity was detected in there. However, after turning into cotyledonary leaves, important levels of gene expression were noted. Taken together, these results suggest that the patterns of alkaloid tissue distribution are established from very early development, and might require transport systems.
\end{abstract}

Keywords: Argemone mexicana; berberine; benzylisoquinoline alkaloids; sanguinarine

\section{Introduction}

Alkaloid synthesis is a complex process which is frequently associated with tissue and organ constitution. It can involve the participation of two or more organs, e.g., roots and aerial parts [1]; the development of specialized appendices and cell structures, such as trichomes and lactiferous cells [2,3]; and the formation of internal organelles, such as alkaloid accumulation vesicles [2]. Alkaloid synthesis in Argemone mexicana L. presents some of these requirements. The Argemone genus belongs to the Papaveraceae family. It includes over 25 species, all of them producers of the tyrosine-derived alkaloids known as benzylisoquinoline alkaloids (BIA's) [4]. The main BIA's identified in A. mexicana include the benzophenanthridine (sanguinarine and dihydrosanguinarine) and the protoberberine (berberine) groups [5] (Figure 1). In mature plants, these alkaloids present a clear tissue distribution, with sanguinarine being restricted to the roots and mature seeds, whereas berberine can be found in both aerial and underground parts, but it is absent in mature seeds [6]. Interestingly, the transcriptional activity related to the synthesis of these alkaloids in tissues of fully developed mature plants does not always match the presence of their products, suggesting long distance transport or communication between the above- and underground parts [6]. Both berberine and sanguinarine biosynthesis are activated from the early stages of seedling unfolding, and tissue spatial distribution is also set from these initial stages of development [7]. Interestingly, newly displayed cotyledons from developing seedlings have been able to accumulate important amounts of sanguinarine, although most of it was found in the seed coats. Because a limited biosynthetic capacity was detected in these tissues, this alkaloid was likely inherited from the original seed [7]. Sanguinarine increased markedly in seedlings later, upon the formation of the first pair of meristematic leaves, coinciding with the expression of biosynthetic genes [7]. This suggests 
that sanguinarine could be formed in the early developmental phases, contrasting with what occurs in mature plants [6,7]. It is noteworthy that sanguinarine synthesis in the aerial parts of $A$. mexicana was also detected in rootless in vitro shoots. However, once roots have formed, this organ turns into the only accumulation site [8,9]. On the other hand, berberine was mainly accumulated in the aerial parts of developing seedlings, regardless of the low expression of the biosynthetic genes in them, compared to that detected in radicles [7].

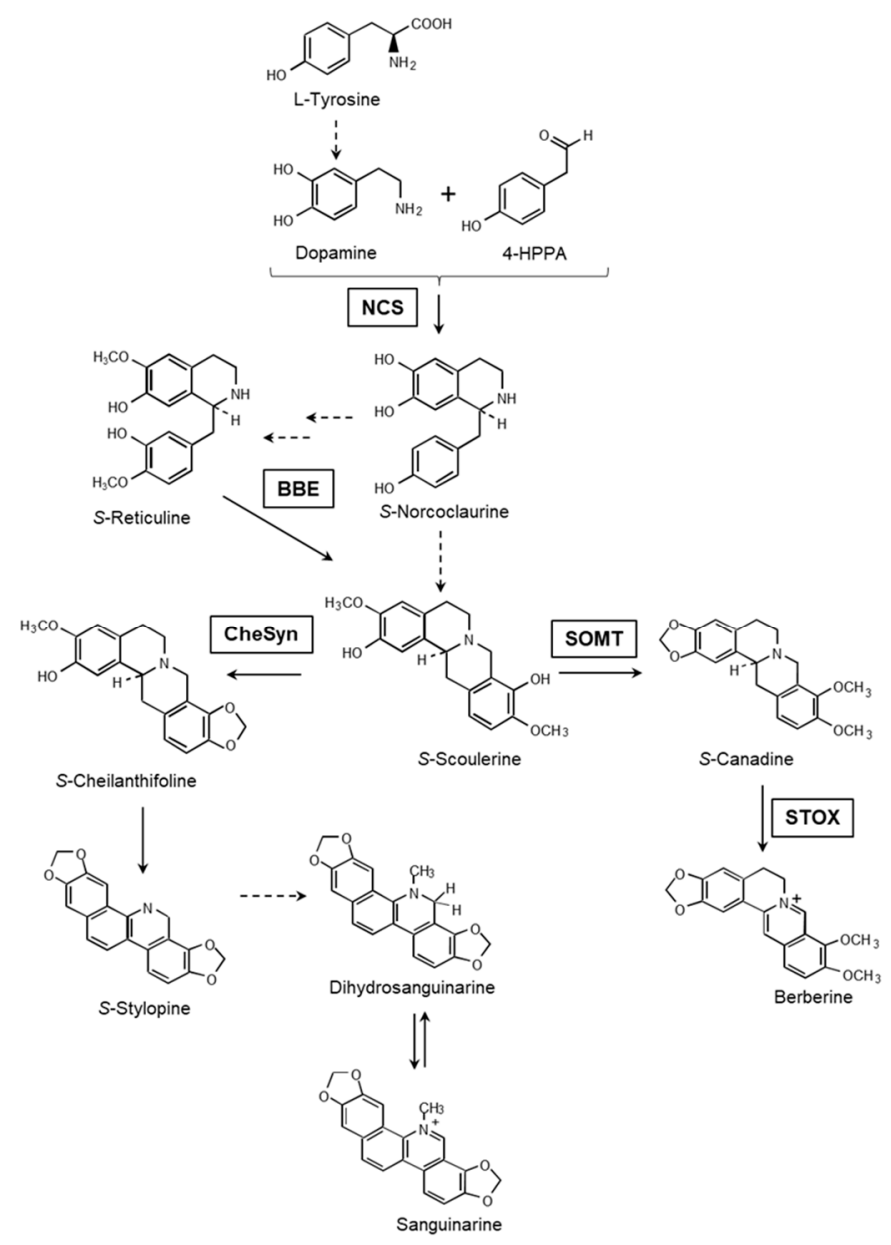

Figure 1. Condensed view of sanguinarine and berberine biosynthesis. The dashed lines represent multiple reactions. NCS, norcoclaurine synthase; $B B E$, berberine bridge enzyme; SOMT, $S$-scoulerine O-methyltransferase; STOX, tetrahydroprotoberberine oxidase; CYP71914 (ChESYN), $S$-cheilanthifoline synthase.

We are interested in establishing how early the actual onset of alkaloid synthesis occurs during the process of seedling germination in Argemone. Here, we followed the alkaloid synthesis throughout the very early stages of germination and organ development in A. mexicana hypocotyls. The data show that both berberine and sanguinarine synthesis in radicles is initiated once they emerge from seed cotyledons.

\section{Results}

Tissues of A. mexicana can accumulate both berberine and sanguinarine, depending on their morphogenic development. During early germination, alkaloid synthesis was associated to cotyledon display [7]. Here, we analyzed the previous events, i.e., radicle protrusion, hypocotyl development and apical hook formation. In order to confirm previous results, unfolding seedlings were also analyzed. Besides the alkaloid accumulation, the biosynthetic pathway was also monitored. This was achieved by estimating the transcript levels for NCS and BBE, which are involved in the early common reactions; SOMT and 
STOX, which are committed to berberine formation; and ChESYN (CYP719A14), which is involved in sanguinarine synthesis (Figure 1).

\subsection{Hypocotyl and Seedling Development}

Hypocotyls and seedlings were collected at different stages of development, registering the time, in days, after sowing. For this work, hypocotyls (stage H) were defined as those formed after radicle protrusion and before apical hook formation. Five developmental stages, named $\mathrm{H} 1$ to $\mathrm{H} 5$, were analyzed. $\mathrm{H} 1$ corresponded to seeds with cracked coats but prior to radicle protrusion; $\mathrm{H} 2$ and $\mathrm{H} 3$ were, respectively, those at radicle development and initial hypocotyl development, whereas $\mathrm{H} 4$ and $\mathrm{H} 5$ represented apical hook formation and hook straightening (Figure 2A). The seedling stage (stage S) was that starting with cotyledon unfolding, and it was also followed through five stages: S1 to S5. S1 and S2 corresponded to the cotyledonary leaves up to the detection of apical meristem sprouting; S3 and $\mathrm{S} 4$ corresponded to the expansion of the first pair of true leaves, whereas the formation of the second pair corresponded to S5 (Figure 2B).

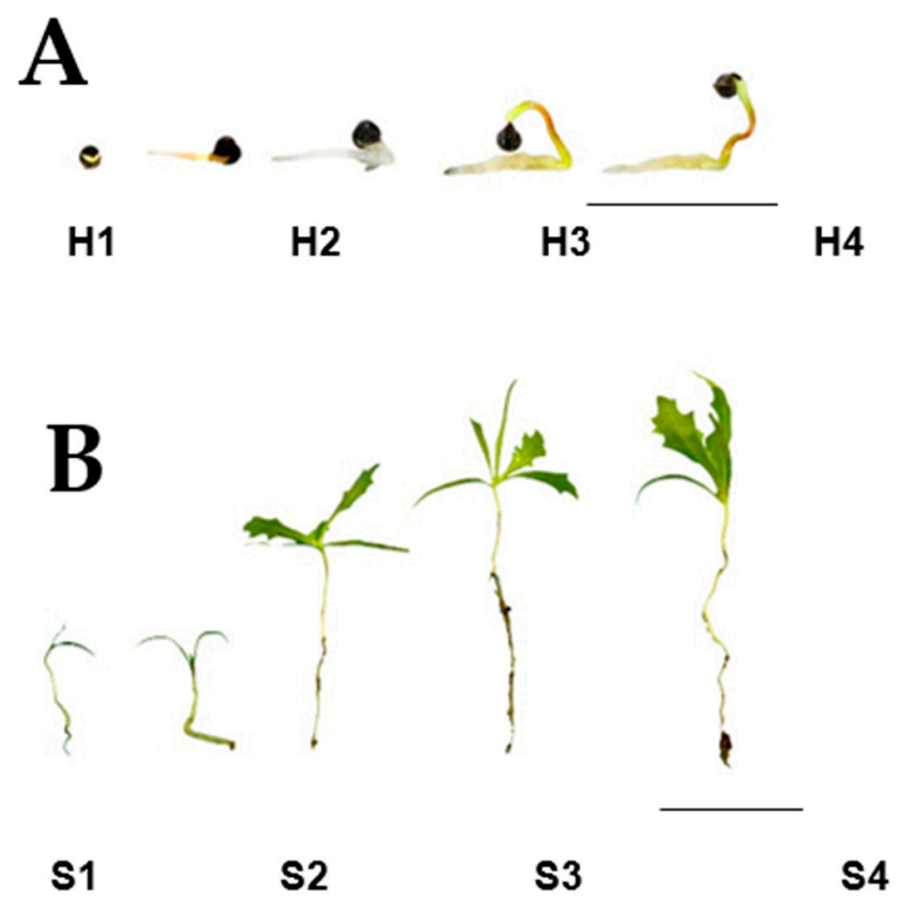

Figure 2. Aspects of A. mexicana hypocotyls (A) and seedlings (B). The scale bars represent $1 \mathrm{~cm}$ in each case.

\subsection{Alkaloid Synthesis at the Hypocotyl Stage}

In mature seeds, prior to germination, sanguinarine is accumulated in high amounts (ca. $1200 \mu \mathrm{g} / \mathrm{g} \mathrm{DW}^{-1}$ ), mainly in the seed coats, where it remains during seedling emergence [7]. The alkaloid amounts recovered from uncoated seed cotyledons represent a minor proportion of the seed's total alkaloid content [7]. Hence, because the seed coats were removed before analysis, the contents detected in cotyledons from hypocotyls represented the newly formed alkaloids. The sanguinarine contents in cotyledons increased continuously through stages $\mathrm{H} 2$ to $\mathrm{H} 4$ (Figure $2 \mathrm{~A}$ ) to a maximum (near to $550 \mu \mathrm{g} / \mathrm{g} \mathrm{DW}^{-1}$ ), shortly before hook straightening (Figure $3 \mathrm{~A}$ ). These maximal sanguinarine values represented ca. $50 \%$ of those found in the complete seeds, prior to germination [7], and they occurred once the apical hook was well defined (H4; Figure 2A). It is noteworthy that sanguinarine markedly decreased when the cotyledons straightened, just before the cotyledonary leaves' emergence (H5; Figure 2A). Hook straightening resulted in the loss of the sanguinarine accumulation in these parts (Figures $2 \mathrm{~A}$ and $3 \mathrm{~A}$ ). In radicles, sanguinarine could be barely detected until stage $\mathrm{H} 4$, reaching its maximum before the cotyledon leaves 
were displayed at $\mathrm{H} 5$ stage (Figures $2 \mathrm{~A}$ and $3 \mathrm{~A}$ ). The berberine contents in the cotyledons at the hypocotyl stage remained at low levels $\left(<80 \mu \mathrm{g} / \mathrm{g} \mathrm{DW}^{-1}\right)$, and were detected at the beginning of hook formation ( $\mathrm{H} 4$ and onwards; Figure 3A). Contrastingly, considerable amounts (ca. $400 \mu \mathrm{g} / \mathrm{g} \mathrm{DW}^{-1}$ ) were observed in the radicles, as soon as they emerged (H2), and remained in such values up to the hook straightening (H5; Figure 3A).
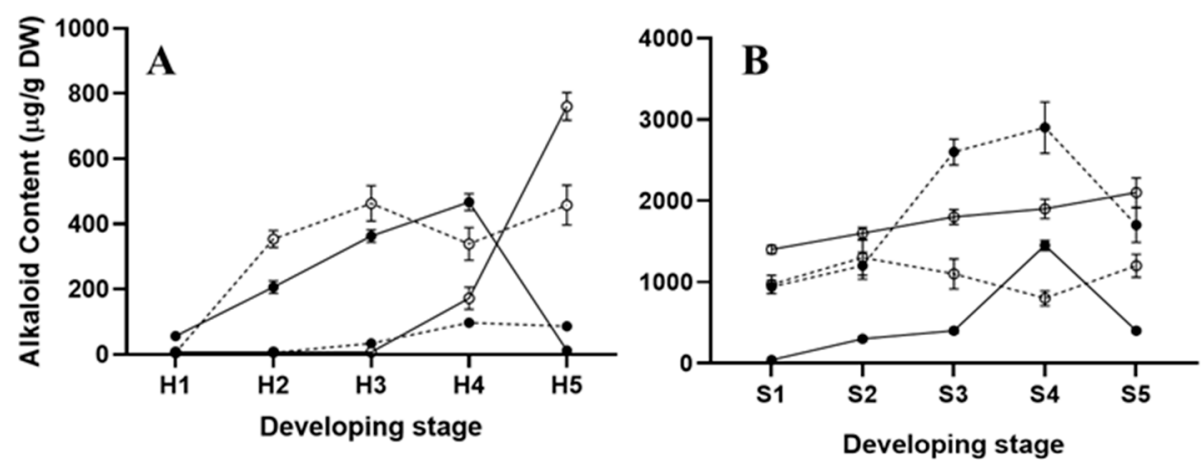

Figure 3. Alkaloid contents in different tissues in the hypocotyl (A) and seedling (B) throughout different developmental stages in A. mexicana. The developmental stages labeled 1 to 5 correspond to H1 to H5 (A) or S1 to S5 (B), as described in the text. The closed and open circles represent the alkaloid contents in cotyledons and radicles, respectively, whereas the continuous and dashed lines stand for sanguinarine and berberine, respectively. The average of triplicates with the standard deviation is given.

Transcriptional activity related to alkaloid biosynthesis was detected in both the hypocotyls' cotyledons and radicles (Figure 4). Markers associated to the early reactions (NCS and BBE) were detected as a single isoform, equally in cotyledons and radicles (NCS-1 and $B B-1$; Figure $4 A, B)$. However, differences were noticed in markers corresponding to specific alkaloids, because neither those associated to sanguinarine (ChESYN) nor berberine (SOMT and STOX) were observed in cotyledons. In contrast, both of them could be detected in radicles (Figure 4C). In cotyledons, NCS-1 and ChESYN transcripts followed the same trend as the sanguinarine accumulation, i.e., a continuous increase throughout the different developmental stages, up to hook formation (H4), followed by an abrupt decrease after straightening (H5) (Figure 2A). Conversely, BBE-1 was maintained at similar levels from the very early stages through to $\mathrm{H} 5$. These results suggest that the sanguinarine accumulated in the cotyledons could have been imported from developing hypocotyls. Correspondently to the lack of significant amounts of berberine in the cotyledons (Figure 3A), neither the expression of SOMT nor of STOX were detected in this part. In hypocotyls, the expression of only the NCS-1 isoform was observed, and it increased as the development proceeded (Figure 4B). Interestingly, a relatively high expression was detected at the medium stages ( $\mathrm{H} 2$ and $\mathrm{H} 3$, corresponding to the early and late hook formation; Figure 2A), and seemed related to berberine synthesis, because no sanguinarine was noticed in the radicles at such periods (Figure 3A). ChESYN was also barely detected at the early developmental stages in hypocotyls, showing a noticeable increase simultaneously with the sanguinarine accumulation (Figures $3 \mathrm{~A}$ and $4 \mathrm{C}$ ). Although it was at low levels, the expression of SOMT and STOX (Figure 4C) coincided with the presence of the alkaloids. This suggests that, as a difference to cotyledons, the alkaloids detected in hypocotyls could have been formed in the same tissue. 

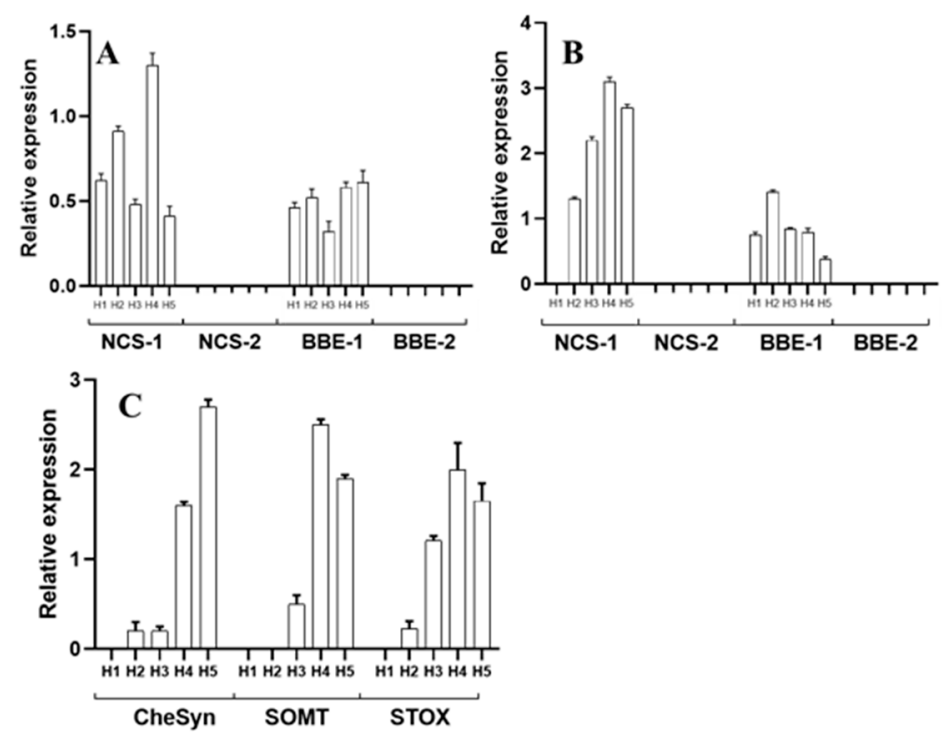

Figure 4. Relative abundance of the transcript involved in alkaloid biosynthesis in $A$. mexicana hypocotyls. The developmental stages correspond to $\mathrm{H} 1$ to $\mathrm{H} 5$, as shown in Figure 2A. (A,B) are the relative transcript abundance for NCS-1 and $B B E-1$ in the cotyledons and radicles, respectively. NCS-2 and BBE-2 were below the detection limits. (C) is the relative transcript abundance for SOMT, STOX and CYP71914 (ChESYN) in the radicles (the levels in cotyledons were undetectable). The average of triplicates with the standard deviation is given. ACTIN was used as the reference gene.

\subsection{Alkaloid Synthesis at the Seedling Stage}

The alkaloid amounts in the seedlings were augmented notably once the cotyledonary leaves were open (Figure 3B). However, low sanguinarine amounts were observed, except at the S4 stage, when the formation of the second pair of meristematic leaves occurred (Figures $2 \mathrm{~B}$ and $3 \mathrm{~B}$ ). In the radicles, sanguinarine presented a minor increase from the amounts detected prior to the cotyledon opening, and remained between 1400 and $2000 \mu \mathrm{g} / \mathrm{g} \mathrm{DW}^{-1}$ throughout the analyzed period (Figure 3B). In contrast, the transition from the $\mathrm{H}$ to $\mathrm{S}$ stage produced a marked increase in berberine accumulation (from less than 80 up to $1000 \mu \mathrm{g} / \mathrm{gDW}^{-1}$ between the $\mathrm{H} 5$ and S1 stages; Figures $2 \mathrm{~B}$ and $3 \mathrm{~B}$ ). The maximal values were observed once the meristematic leaves were formed (S3 and S4; around $3000 \mu \mathrm{g} / \mathrm{gDW}^{-1}$ ) and, although it decreased afterwards, it was kept above $1400 \mu \mathrm{g} / \mathrm{gDW}^{-1}$ (Figure 3B). In the radicles, the berberine accumulation followed a similar trend to that of sanguinarine, showing little variation throughout development (ca. $1000 \mu \mathrm{g} / \mathrm{g} \mathrm{DW}^{-1}$; Figure 3B). In this way, berberine was the main alkaloid in the aerial parts once they were formed.

Contrasting with the hypocotyls, the transcriptional activity related to alkaloid synthesis was equally detected in both the aerial and underground parts of the seedlings. Moreover, the two isoforms for NCS and BBE were equally detected in the aerial parts and radicles, although no differential expression patters were noticed for any of these isoforms. As a general trend, a developmentally related increase in expression was noticed for transcripts corresponding to both enzymes (Figure 5A,B). Interestingly, ChESYN expression was observed in the cotyledonary leaves, even when no significant accumulation of sanguinarine was detected in these parts (Figure 5C). The expression levels were similar to those in the radicles (Figure 5D), which presented much higher sanguinarine amounts (Figure 3B). These data suggest that the cotyledonary leaves could contribute to the sanguinarine levels found in the radicles. Contrastingly, the expression of berberineformation-related genes (SOMT and STOX) was detected in high levels throughout the analyzed developmental stages in both the aerial parts (Figure 5C) and radicles (Figure 5D), with a good correspondence to the alkaloid amounts detected in these tissues. 

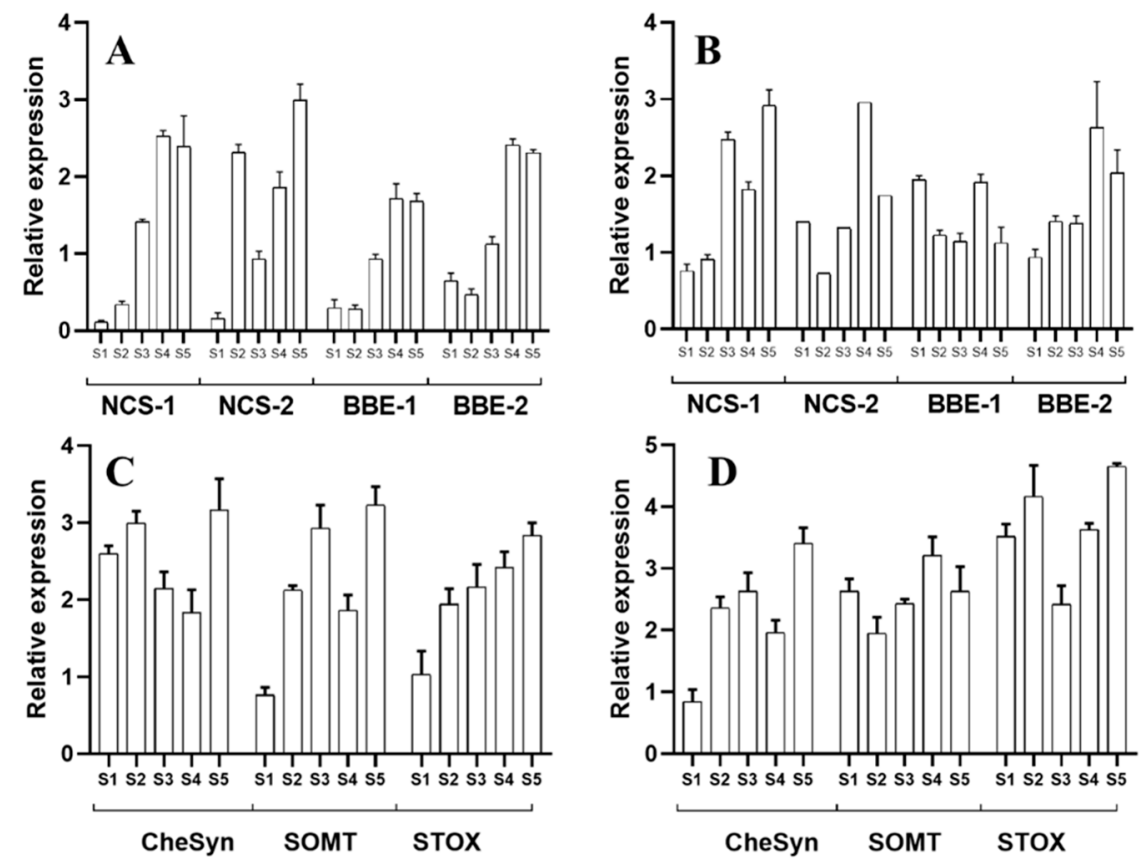

Figure 5. Relative abundance of the transcript involved in alkaloid biosynthesis in A. mexicana hypocotyls. The developmental stages correspond to S1 to S5, as shown in Figure 2B. (A,B) are the relative transcript abundance for NCS and BBE in cotyledons and radicles, respectively. $(\mathbf{C}, \mathbf{D})$ are the relative transcript abundance for SOMT, STOX and CYP71914 (ChESYN). The average of triplicates with the standard deviation is given. ACTIN was used as the reference gene.

\section{Discussion}

Germination and hypocotyl/seedling development represent the interpretation of the plant's master plan imprinted in the embryo. Bipolar embryo development sets the patterns for aerial and underground organ formation [10,11]. In natural conditions, the early stages of seed germination take place underground, resulting in hypocotyls with an apical hook and closed cotyledons. Once hypocotyls emerge from the soil, light triggers hook straightening and the displaying of green cotyledons, leading to a switch from heterotrophy to autotrophy. Such a modification involves both morphological and metabolic changes [12], including the onset of secondary metabolism [13].

The alkaloid synthesis in A. mexicana occurred from the very early stages of seedling germination, once the hypocotyls protruded (Figures 2-5). In the very early phases, the cotyledons from hypocotyls accumulated sanguinarine in higher amounts than the radicles (Figure 3A), and this coincided with the transcriptional activity detected in radicles (Figure 4). The sanguinarine in the radicles was augmented only when the cotyledons were ready to open, and kept on increasing during the aerial part development (Figure 3A,B). This suggests the operation of transport systems, moving alkaloids from the hypocotyl radicles to the cotyledons. Interestingly, the aerial parts started expressing ChESYN even when no sanguinarine was accumulated in them (with the exception of the S4 peak). Taken together, these results suggest that, in the very early stages of germination, radicles might carry out most of the sanguinarine synthesis for exportation to the cotyledons. However, once the cotyledons expanded, radicles seem to store the formed sanguinarine, in addition to some provided from the aerial parts. This does not seem to be the case for berberine, which coincided both in the cotyledons and radicles with the SOMT transcripts (Figures 2-5). Interestingly, during the early stages of development, prior to the meristematic leaves' formation, no vascular tissues were formed [13]. This suggests that alkaloids could move between tissues using a cell-to-cell transport mechanism. Such a type of mechanism has been described for other alkaloids, such as the pirrolizidines [14]. In fact, a transcriptome from developing seedlings of $A$. mexicana $[6,7]$ revealed the presence of 
several putative $\mathrm{ABC}$ proteins, which have been involved in benzylisoquinoline alkaloid transport [15].

\section{Materials and Methods}

\subsection{Plant Materials}

The seed coats were mechanically detached from hypocotyls for the alkaloid analysis. The samples were freeze-dried and ground to a fine powder using a mechanical mill (IKA Tube Mill CS001; Wilmington NC). The alkaloids were extracted from $50 \mathrm{mg}$ powdered tissues in $10 \mathrm{~mL} 0.5 \%$ hydrochloric acid in methanol, as previously described [16]. Briefly, the powders were incubated for two hours with gentle shaking at room temperature, and the debris was separated by filtration. Next, $1 \mathrm{~mL}$ aliquots were centrifuged for the further remotion of the tissue remains, and were used for the chromatographic analysis. The alkaloids were separated by TLC, using a mixture of n-butanol:water: $\mathrm{NH}_{4} \mathrm{OH}(8: 1: 1)$, or benzene:ethanol (9:1), for berberine $\left(R_{f} 0.29\right)$ and sanguinarine $\left(R_{f} 0.53\right)$ detection, respectively. After the chromatographic separation, the alkaloids were quantified by in situ fluorescence in a Camag TLC Scanner 4 (Muttenz Switzerland) controlled by the WinCATS 1.4.10 planar chromatography manager [16]. The analysis was performed in triplicates.

\subsection{Nucleic Acid Analysis}

The total RNA was extracted as described elsewhere [17]. The analyzed genes corresponded to common biosynthetic reactions, as well as being committed to the specific synthesis of berberine and sanguinarine (Figure 1). Norcoclaurine synthase (NCS-1 and -2; EU881891 and EU881893), the berberine bridge enzyme (BBE-1 and -2; EU881889 and EU881890), scoulerine O-methyltransferase (SOMT; KT984756), STOX (tetrahydroprotoberberine oxidase; HQ116698) and S-cheilanthifoline synthase (ChESYN; CYP719A14; EF451152) were analyzed to monitor the alkaloid biosynthetic pathways (Figure 1). NCS and $B B E$ represent the early and common biosynthetic reactions; SOMT and STOX represent those committed to berberine, whereas ChESYN is specific to sanguinarine formation $[7,18,19]$. Specific oligonucleotide primers [7] were used to analyze the transcripts relative abundance by quantitative real time PCR (qRT-PCR). See Appendix A for the primer sequences. The first strand CDNA was synthesized using M-MLV reverse transcriptase (Invitrogene, Carlsbad, CA, USA) according to standard procedures. The qRT-PCR was performed using an Eco ${ }^{\mathrm{Tm}}$ Real Time PCR System (Illumina, San Diego, CA, USA). The reaction mixture $(20 \mu \mathrm{L})$ contained $10 \mu \mathrm{L}$ SYBR Green Mastermix (Applied Biosystems, United Kingdom), $10 \mu \mathrm{M}$ of each primer, and $5 \mathrm{ng}$ of the RT products. A 35-cycle program was used $\left(50,30\right.$, and $30 \mathrm{~s}$ at 95,60 , and $60^{\circ} \mathrm{C}$ for the DNA denaturation, primer alignment and amplification, respectively). The gene transcripts were quantified using the cycle threshold value $(\mathrm{Ct})$, using ACTIN as a reference [20]. Prior to performing the qPCR, amplicon's identity was verified by sequencing it after the standard PCR with the same set of primers. The analysis was performed in triplicates.

\section{Conclusions}

Taken together, these results suggest that the patterns of alkaloid tissue distribution are established from very early development, and might require transport systems.

Author Contributions: Conceptualization, J.X.-T. and F.V.-F.; methodology, J.X.-T. and Y.T.-O.; formal analysis, J.X.-T., Y.T.-O., M.M.-G., J.A.M.-S.; writing—original draft preparation, J.X.-T., Y.T.-O., M.M.G., J.A.M.-S. and F.V.-F.; writing-review and editing, supervision, project administration and funding acquisition, F.V.-F. All authors have read and agreed to the published version of the manuscript.

Funding: This work was supported by CONACYT (National Council for Science and Technology, Mexico), grant CB-2016-0285887.

Institutional Review Board Statement: Not applicable.

Informed Consent Statement: Not applicable. 
Data Availability Statement: All of the data are publicly available in the databases mentioned.

Acknowledgments: The authors wish to thank María de Lourdes Miranda-Ham for her critical review, and Victor Manuel Carballo-Uicab for his assistance in the figure editing.

Conflicts of Interest: The authors declare no conflict of interest.

\section{Appendix A}

The specific primers were taken from [6,7]. Accession numbers: NCS-1 and -2 (norcoclaurine synthase), EU881891 and EU881893, respectively; BBE-1 and -2 (berberine bridge enzyme), EU881889 and EU881890, respectively; SOMT (scoulerine O-methyltransferase), KT984756; STOX (tetrahydroprotoberberine oxidase), HQ116698; and ChESYN/CYP719A14 (cheilanthifoline synthase), EF451152.

Table A1. Sequence of the primers used for qRT-PCR.

\begin{tabular}{ccl}
\hline Gene & Direction $\mathbf{1}^{2}$ & \multicolumn{1}{c}{ Sequence $\mathbf{( 5}^{\prime}-\mathbf{3}^{\prime} \mathbf{)}$} \\
\hline ACTIN & Fwd & CACIACTACTGCTAAACGGGAAA \\
& Rvs & ACATCTGCTGGAAGGTGCTG \\
\hline FyDC & Fwd & GTTGAACCAGGTTATTTACG \\
& Rvs & CGTATTCTTTCGCAACCTC \\
\hline FCS-1 & Fwd & CATCGCTAATTACGTTCTCAAGAATCA \\
& Rvs & ATAGTAGTACATGGAATTACCTGGATGGGA \\
\hline FCS-2 & Fwd & CGTACCATTGAAATCCATGTCAGAA \\
& Rvs & CATCGGACGGTAATTACCCATG \\
\hline BBE-1 & Fwd & CATCTTTGTTCATCATCATCTTCTTCTTCTTCTT \\
& Rvs & GATCCTCTTGTGCAACATCTAACGGT \\
\hline BBE-2 & Fwd & CTCATCTTTGTTCATCTTCTTTTCTGTGC \\
& Rvs & GATCCTCTTGTGCAACATCTAACGGT \\
\hline CHESYN & Fwd & AGGTCTTCAAGGTGTTGCCC \\
$(C Y P 719 A 14)$ & Rvs & TCTTTTCCCGCCCGTAACAT \\
\hline SOMT & Fwd & ATCCTATCCATGTCTACGAGGGCTATT \\
& Rvs & CCAGTACCACCACCAACATCTAACA \\
\hline STOX & Fwd & GGTTAGGAAATATGGACTTG \\
& Rvs & ATAACATTGCTGGTGAATCT \\
\hline
\end{tabular}

${ }^{1}$ Fwd, forward; Rvs, reverse.

\section{References}

1. Hildreth, S.B.; Gehman, E.A.; Yang, H.; Lu, R.-H.; Ritesh, K.C.; Harich, K.C.; Yu, S.; Lin, J.; Sandoe, J.L.; Okumoto, S.; et al. Tobacco nicotine uptake permease (NUP1) affects alkaloid metabolism. Proc. Natl. Acad. Sci. USA 2011, 108, 18179-18184. [CrossRef] [PubMed]

2. Ziegler, J.; Facchini, P.J. Alkaloid Biosynthesis: Metabolism and Trafficking. Annu. Rev. Plant Biol. 2008, 59, 735-769. [CrossRef] [PubMed]

3. Schilmiller, A.L.; Last, R.L.; Pichersky, E. Harnessing plant trichome biochemistry for the production of useful compounds. Plant J. 2008, 54, 702-711. [CrossRef] [PubMed]

4. Schwarzbach, A.E.; Kadereit, J.W. Phylogeny of prickly poppies, Argemone (Papaveraceae), and the evolution of morphological and alkaloid characters based on ITS nrDNA sequence variation. Plant Syst. Evol. 1999, 218, 257-279. [CrossRef]

5. Rubio-Piña, J.; Vázquez-Flota, F. Pharmaceutical applications of the benzylisoquinoline alkaloids from Argemone mexicana L. Curr. Top. Med. Chem. 2013, 13, 2200-2207. [CrossRef] [PubMed]

6. Vázquez-Flota, F.; Rubio-Piña, J.; Xool-Tamayo, J.; Vergara-Olivares, M.; Tamayo-Ordoñez, Y.; Monforte-González, M.; GuízarGonzález, C.; Mirón-López, G. Tissue distribution of transcripts involved in benzylisoquinoline alkaloid biosynthesis in Argemone mexicana L. (Papaveraceae). Rev. Fitotec. Mex. 2018, 41, 13-21. [CrossRef]

7. Xool-Tamayo, J.F.; Monforte-González, M.; Rubio-Piña, J.; Mirón-López, G.; Vázquez-Flota, F. Early developmental onset of alkaloid biosynthesis in Mexican poppy (Argemone mexicana L.) Papaveraceae. Phytochem. Lett. 2017, 20, 300-305. [CrossRef] 
8. Xool-Tamayo, J.; Serrano-Gamboa, G.; Monforte-González, M.; Mirón-López, G.; Vázquez-Flota, F. Development of newly sanguinarine biosynthetic capacity in in vitro rootless shoots of Argemone mexicana L. Mexican prickly poppy. Biotechnol. Lett. 2017, 39, 323-330. [CrossRef] [PubMed]

9. Monforte-González, M.; Serrano-Gamboa, J.G.; Guízar-González, C.; Miranda-Ham, M.L.; Vázquez-Flota, F.A. Alkaloid synthesis is coupled to shoot morphogenesis in Argemone mexicana L. (Papaveraceae) in vitro cultures. Vitr. Cell. Dev. Biol. Plant 2019, 55, 695-701. [CrossRef]

10. Sullivan, J.; Deng, X.W. From seed to seed: The role of photoreceptors in Arabidopsis development. Dev. Biol. 2003, 260, 289-297. [CrossRef]

11. Harada, J.J. Role of Arabidopsis LEAFY COTYLEDON genes in seed development. J. Plant Physiol. 2001, 158, 405-409. [CrossRef]

12. Yang, N.; Ye, Z.; Liu, J.; Liu, Y.; Chen, Q.; Wang, H.; Guo, X.; Herppich, W.B.; Tang, Z. Network during light-induced cotyledons opening and greening in Astragalus membranaceus. J. Plant Interact. 2020, 15, 358-370. [CrossRef]

13. Hardtke, C.S.; Berlett, T. The Arabidopsis gene MONOPTEROS encodes a transcription factor mediating embryo axis formation and vascular development. EMBO J. 1998, 17, 1405-1411. [CrossRef] [PubMed]

14. Langel, D.; Ober, D.; Pelser, P.B. The evolution of pyrrolizidine alkaloid biosynthesis and diversity in the Senecioneae. Phytochem. Rev. 2011, 10, 3-74. [CrossRef]

15. Shitan, N.; Bazin, I.; Dan, K.; Obata, K.; Kigawa, K.; Ueda, K.; Sato, F.; Forestier, C.; Yazaki, K. Involvement of CjMDR1, a plant multidrug-resistance-type ATP-binding cassette protein, in alkaloid transport in Coptis japonica. Proc. Natl. Acad. Sci. USA 2003, 100, 751-756. [CrossRef] [PubMed]

16. Monforte-González, M.; Guízar-González, C.; Rubio-Piña, J.; Carrillo-Pech, M.; Vázquez-Flota, F. Berberine and sanguinarine quantitation in Argemone mexicana L. (Papaveraceae) tissues by TLC-in situ fluorography. J. Planar Chromatogr. Mod. TLC 2012, 25, 358-360. [CrossRef]

17. Rubio-Piña, J.; Vázquez-Flota, F. Isolation of functional total RNA from Argemone mexicana tissues. Electron. J. Biotechnol. 2008, 11, 1-5. [CrossRef]

18. Chávez, M.L.D.; Rolf, M.; Gesell, A.; Kutchan, T.M. Characterization of two methylenedioxy bridge-forming cytochrome P450dependent enzymes of alkaloid formation in the Mexican prickly poppy Argemone mexicana. Arch. Biochem. Biophys. 2011, 507, 186-193. [CrossRef] [PubMed]

19. Gesell, A.; Díaz-Chávez, M.L.; Kramell, R.; Piotrowski, M.; Macheroux, P.; Kutchan, T.M. Heterologous expression of two FAD-dependent oxidases with (S)-tetrahydroprotoberberine oxidase activity from Argemone mexicana and Berberis wilsoniae in insect cells. Planta 2011, 233, 1185-1197. [CrossRef] [PubMed]

20. Simon, P. Q-Gene: Processing quantitative real-time RT-PCR data. Bioinformatics 2003, 19, 1439-1440. [CrossRef] [PubMed] 